\title{
Survey requirements of the accreditation program after the first round of the national assessment at hospital and compared with the results of it
}

\author{
Meghdad Rahati ${ }^{1}$, Monireh Dehghani Arani' ${ }^{2}, \operatorname{Reza}_{\text {Nanakar }}{ }^{1,}$, Azam Kazemi ${ }^{3}$ \\ ${ }^{1}$ Dept. Management and Health Information Technology, Kashan University of Medical Sciences, Kashan, Iran \\ ${ }^{2}$ Dept. Biostatistics, Shahid Beheshti University of Medicine Sciences, Tehran, Iran \\ ${ }^{3}$ Dept. Nursing, Kashan University of Medical Sciences, Kashan, Iran
}

\section{Email address:}

r_nanakar@yahoo.com (R. Nanakar)

\section{To cite this article:}

Meghdad Rahati, Monireh Dehghani Arani, Reza Nanakar, Azam Kazemi. Survey Requirements of the Accreditation Program after the First Round of the National Assessment at Hospital and Compared with the Results of It. International Journal of Economic Behavior and Organization. Vol. 2, No. 1, 2014, pp. 6-12. doi: 10.11648/j.jjebo.20140201.12

\begin{abstract}
The idea of quality management systems in recent years in the public and governmental sectors have attracted attention of officials and experts .Can say that correct deployment of these systems is one of the most important tools that can assist the governments in this matter, and lead to success. In this regard, We decided to survey requirements of this program in the hospitals. Methods: we survey the comments of 85 managers and supervisors of hospitals with five factors influencing on this program and then compared with the results of national assessment. In a descriptive crosssectional study, we used a five-part questionnaire with 40 Likert-type questions for data collection that Validated with Delphi method. analysis of variance and T-test were used for statistical analysis. Results: among the five factors, Leadership and management $(\% 57,88)$ Organizational structure $(\% 62,03)$ Human resource development $(\% 60,58)$ customer focus $(\% 56,35)$ and Team work $(\% 58,41)$ proved to be acceptable.(with assuming $50 \%$ accepted in the five-factor).There was significant difference among the total means of influencing factors in the studies hospitals(P-value <0.0001) also between Total score (comments of managers and supervisors) and score of national assessment there was no significant differences. $(\mathrm{P}-\mathrm{value}=0.2)$ Conclusion: It can be said that attention of managers to Leadership and management, Organizational structure, Human resource development, Customer focus and Team work will make effective and efficient implementation Accreditation Program.
\end{abstract}

Keywords: Requirements, Accreditation Program, Hospital

\section{Introduction}

Today competition to quality improve service has been known as a key strategic issue for organizations that are active in the service sector. Organizations that have a higher level of quality service, will achieve higher levels of customer satisfaction as a prelude to achieving sustainable competitive advantage(Guo, Duff, \& Hair, 2008) Undoubtedly, create satisfaction in the customers and even enthusiasm in their quality service, in the first stage, requires understanding their needs and desires and then transfer it to a position that goods and services are produced. This is due to increasing complexity of economic system, social and cultural, that does not happen spontaneously, but systematic methods and procedures is needed to change these concepts into organizational process. (ghaderi \& hosseini, 2010).

In this regard, in the health system, most managers and politicians have used various model of evaluation, control and quality improvement to promote their organizations and clients satisfaction. Today, technological advances, growing customer expectations, increased demand, lack of resources, increased competition, and concerns about safety, malpractice and health care system errors has caused to becomes assessment and evaluation strict performance of healthcare organizations more important. (azami, tabrizi, \& etal, 2012) The main elements of the health system is hospitals that Health reform without attending to these and improve their performance would not be possible. So that many assessment and evaluation that is required by the health system, in these centers will rise. (Amerioun, 
Mahdavi, Mamaghani, \& etla, 2011).

One methods of improving quality and safety in health care organization is accreditation, which focuses on continuous improvement, quality improvement, patient safety and staff. Accreditation is a means of systematic evaluation health care providers. Accreditation with use a group of experts in a specialized field, based on codified standards that have been developed to measure quality of patient care, is attempting to evaluate a health care organization and make decisions about their qualifications, to determine reputation, credibility and recognition center that able to perform certain services as standard. (abasi, tavakoli, \& moslehi, 2012).

Accreditation is an independent, voluntary program that at year 1917 in the United States by the Joint Commission on Accreditation (JCAHO) was formed to evaluate the health service provider organizations. International branch of accreditation with the name of International Joint Commission (JCI) began in 1998, to investigate the accreditation requirements at the international level with the formulation and development a group of accreditation standards, Which in 1999 published first edition of the International Standards. (Amerioun , Mahdavi, Mamaghani, \& etal, 2011).

Joint Commission International Accreditation Standards worldwide, are unique tools that are designed to measure the quality of patient care. Research in this field suggests that the international standards accreditation provides a framework that can be used as a common model to assess health care in the World. (ahmadi, khoshgam, \& mohamad pour, 2008).

From the 1950s until the early 1990s, there was only accreditation programs in developing countries but increasing need to improve quality of health care and increasing medical errors has been caused dramatic expansion in recent years. So that in the 1990s some developing countries also have been implemented, and until 2004, almost 60 countries either performer or ongoing to creating the process of national accreditation program for health care. (Korrani \& Mahdavi, 2008) Implementation of accreditation confirmed as a comprehensive model by the World Health Organization in 2003, and as a protective factor be known for evaluation hospitals performance. (Veillard, Champagne, \& etal, 2005).

In Iran to 2012, Hospital evaluation based on the standards of the Ministry of Health and hospital grade was determined. In this regard, studies indicate that $\mathrm{MOH}$ standards were not very comprehensive. However, with these standards and obtaining grades 1 and 2, some hospitals were lacking quality and efficiency. (ahmadi, khoshgam, \& mohamad pour, 2008) (Ministry of Health., 2007) (Tofighi, 2002) But in recent years the Ministry of Health and Medical Education with continuous efforts and long-term planning, has provided the context for Implementation of program accreditation in 2013.

In this regard, lack of previous experience in this field and despite passing one year since implementation program in hospitals of Iran no any assessment has been done to determine status and success of the project. Therefore, the aim of this research is to enhance the efficiency and effectiveness of the program through the review driving and preventing factors in of the project.

\section{Methods}

This research is a cross - sectional study, which was done in 2013. It was designed to investigate requirements of the accreditation program after the first round of the national assessment in hospitals of Kashan University of Medical Sciences and compared with the results of assessment.

Tools for data collection is questionnaire, which set in 5 parts (40 questions), included Leadership and management, Organizational structure, Human resource development, Customer focus and Teamwork. This questionnaire based on a Likert scale rating. (The range of it between 1 and 5)The scores are categorized as follows:

Score 1: very low

Score 2: low

Score 3: Medium

Score 4: High

And score 5 as much.

For scientific validity of Questionnaire were used content validity. Thus, through study of books and internet search and using experts opinions, required reform were applied in the questionnaire.(Delphi-like method) and final questionnaire were prepared. Number of 85 questionnaires were distributed between managers and supervisors of hospitals and then collected.

For data analysis, descriptive and analytical statistical were used. Thus, data in the frequency distribution tables were classified and index in the descriptive statistics included mean and standard deviation were used. T test and analysis of variance (ANOVA) were used for statistical analysis. $\mathrm{T}$ test used to compare the results of the questionnaire with the evaluation scores and ANOVA used to compare the mean scores of each hospital. This test was estimated with $5 \%$ error. Spss software were used for data analysis.

By determining the highest and lowest mean score for each factor, divided hospitals, in addition to determine status of each hospitals studied, determined rank of each factor as well.

\section{Results}

Based on data obtained from this study, demographic information of population are shown in Table one. $34.1 \%$ of the participants were female and $65.9 \%$ were male.78 persons $(91.7 \%)$ were aged 40-20.The most frequent participants was BA degree $(n=70,82.4 \%)$ and the lowest frequency was related to the diploma $(n=2,4 / 2 \%)$. Also work Experience of participants in this study were distributed equally between 6-12,12-18 and above 18 years. Complementary information is given in Table1. 
Table 1. Demographic characteristics of the study population.

\begin{tabular}{cccc}
\hline Demographic & Frequency & Frequency & percentage \\
\hline \multirow{2}{*}{ sex } & men & 29 & 34.1 \\
& women & 56 & 65.9 \\
age & $20-30$ & 6 & 7.1 \\
& $30-40$ & 47 & 55.2 \\
& $40-50$ & 31 & 36.5 \\
& Up 50 & 1 & 1.2 \\
Degree & Diploma & 9 & 13 \\
& Bachelor & 70 & 82.4 \\
& Masters & 4 & 4.7 \\
& To 6 & 7 & 8.2 \\
& $6-12$ & 30 & 35.3 \\
& $12-18$ & 24 & 28.2 \\
& Up18 & 24 & 28.2 \\
\hline
\end{tabular}

Findings from this study, accordance with Table 2, shows the mean scores in "Management and leadership" with a standard deviation $(0.5)$ is equal to $57.88 \%$.

The mean scores in the "Organizational structure" with a standard deviation (0.19) is equal to $62.03 \%$.

The mean scores in the "Human resource" development with a standard deviation (0.17) is equal to $60.85 \%$

The mean scores in the "Customer Focus "with a standard deviation $(0.1)$ is equal to $56.35 \%$.

The mean scores in the "Team work" with a standard deviation ( 0.29 is equal to $58.41 \%$.

The factor of "Organizational structure" compared with other factors, has the highest ranking with mean $62.03 \%$ and the factor "Customer Focus" has the lowest ranking.
Table 2. Summary of indicators related to program accreditation and chisquare test results.

\begin{tabular}{clcc}
\hline Row & Variables & Mean & $\begin{array}{c}\text { Standard } \\
\text { deviation }\end{array}$ \\
\hline 1 & Leadership and management & 57.88 & 0.5 \\
2 & organizational structure & 62.03 & 0.19 \\
3 & human resource development & 60.85 & 0.17 \\
4 & Customer Focus & 56.35 & 0.10 \\
5 & Teamwork & 58.41 & 0.29 \\
\hline
\end{tabular}

The mean and standard deviations related all questions described briefly in Table3.

As can be seen in factor "Leadership and management" the highest ranking related to "Develop and approve Roadmap for implement the program" and the lowest ranking related to "Proper incentive mechanism for teams and staff in the program" and "Provide the human and financial resources to perform program".

In factor "Organizational structure" the highest ranking related to "Requirement rules and regulations for the support of program" and the lowest ranking related to "Delegation to employees in problem solving" and" Formulation of hospital structure according values.

In factor "Human resource development" the highest ranking related to "establish review and identifying needs training systems" and the lowest ranking related to "Allocation resources to educational requirements" and" Consider to valuable opinions and ideas of employees".

In factor" Customer Focus" the highest ranking related to "Measure level of patients' satisfaction" and the lowest ranking related to "Planning for contact and communicate with clients" and "Planning for applying comments of the customers".

In factor "Teamwork" the highest ranking related to "Determine scope authority of group and duties and the lowest ranking related to "Use of quality control group for solve quality problems" and " Encourage Individuals For teamwork by evaluation system ".

Complementary information is given in Table3.

Table 3. Mean and standard deviation of questions related internal issues of hospital.

\begin{tabular}{|c|c|c|c|c|}
\hline & Row & Subject & $\mathbf{M}$ & S.D \\
\hline \multirow{8}{*}{$\begin{array}{l}\text { Leadership and } \\
\text { management }\end{array}$} & 1 & Commitment of Hospital managers to implement program. & 3.35 & 1.35 \\
\hline & 2 & Develop and approve roadmap for implement the program. & 3.54 & 1.11 \\
\hline & 3 & Identify and prioritize quality improvement fields & 3.37 & 1.12 \\
\hline & 4 & Provide human and financial resources to perform program. & 2.23 & 1.07 \\
\hline & 5 & Proper incentive mechanism for teams and staff in the program. & 2.14 & 1.13 \\
\hline & 6 & Coherently follow up quality improvement activities. & 2.99 & 1.21 \\
\hline & 7 & Sufficient authority to department responsible for the program. & 3.16 & 1.00 \\
\hline & 8 & Benchmarking from Superior hospital for quality. & 3.05 & 1.08 \\
\hline \multirow{8}{*}{$\begin{array}{l}\text { organizational } \\
\text { structure }\end{array}$} & 9 & Explain goals, mission and policies for employees. & 3.30 & 1.02 \\
\hline & 10 & Movement hospital towards goals, mission and policies. & 3.20 & .98 \\
\hline & 11 & Alignment goals and objectives of hospital and staff in the program. & 3.28 & 1.04 \\
\hline & 12 & Formulation hospital structure according values. & 2.96 & 1.13 \\
\hline & 13 & Delegation to employees in problem solving. & 2.87 & 1.06 \\
\hline & 14 & Determine responsibilities and duties of individuals to implement the program. & 3.37 & 1.07 \\
\hline & 15 & Requirement coordination between departments and units to run the program. & 3.18 & 1.04 \\
\hline & 16 & Requirement rules and regulations for the support of the program. & 3.40 & .99 \\
\hline \multirow{2}{*}{$\begin{array}{c}\text { human resource } \\
\text { development }\end{array}$} & 17 & Consider to valuable opinions and ideas of employees. & 3.04 & .93 \\
\hline & 18 & Informing employees timely and adequate. & 3.25 & 1.08 \\
\hline
\end{tabular}




\begin{tabular}{|c|c|c|c|c|}
\hline & Row & Subject & $\mathbf{M}$ & S.D \\
\hline \multirow{14}{*}{ Customer Focus } & 19 & Identification Internal communications to staff. & 3.16 & .92 \\
\hline & 20 & formulation and announcement performance evaluation Indicators & 2.98 & 1.04 \\
\hline & 21 & Establish review and identifying needs training systems. & 3.35 & 1.02 \\
\hline & 22 & Organize training program with the training needs. & 3.31 & .91 \\
\hline & 23 & Allocation resources to educational requirements. & 2.92 & .95 \\
\hline & 24 & Planning for understanding concepts and philosophy of program accreditation. & 2.99 & 1.00 \\
\hline & 25 & Planning for contact and communicate with clients. & 2.91 & .98 \\
\hline & 26 & Give necessary training to clients for deliver services. & 3.17 & .97 \\
\hline & 27 & Planning for applying comments of customers. & 2.97 & 1.01 \\
\hline & 28 & Analyze complaints from consumers. & 3.22 & 1.05 \\
\hline & 29 & Track and trace provided services to clients. & 3.16 & 1.13 \\
\hline & 30 & Measure level of patients' satisfaction. & 3.31 & 1.07 \\
\hline & 31 & Identification factors influencing patients' satisfaction. & 3.05 & .92 \\
\hline & 32 & Determining Optimal service delivery methods for clients. & 3.22 & .91 \\
\hline \multirow{8}{*}{ Teamwork } & 33 & Having working group with structure and clear objectives. & 3.41 & .99 \\
\hline & 34 & Meetings of working groups on specific period. & 3.51 & 1.04 \\
\hline & 35 & Determine scope authority of group and duties. & 3.63 & .78 \\
\hline & 36 & Define indicators for assessing performance of teams. & 3.23 & .91 \\
\hline & 37 & Use of quality control group for solve quality problems. & 2.84 & 1.06 \\
\hline & 38 & Use of working groups to communicate and interact with clients. & 2.91 & .95 \\
\hline & 39 & Appropriate and favorable environment for team activities & 3.00 & 1.05 \\
\hline & 40 & Encourage Individuals For teamwork by evaluation system. & 2.92 & 1.02 \\
\hline
\end{tabular}

According to Table4, for compare comments of managers and supervisors in the hospitals and compare between total score and percent of the national accreditation program standards were used ANOVA and t test orderly.

Table 4. Mean and standard deviation factor and Percent of standards (national assessment), Separation of the studied hospitals.

\begin{tabular}{|c|c|c|c|c|c|c|c|c|c|c|c|c|c|}
\hline \multirow[t]{2}{*}{$\begin{array}{l}\text { category } \\
\text { Hospital }\end{array}$} & \multicolumn{2}{|c|}{$\begin{array}{c}\text { Leadership and } \\
\text { management }\end{array}$} & \multicolumn{2}{|c|}{$\begin{array}{l}\text { organizational } \\
\text { structure }\end{array}$} & \multicolumn{2}{|c|}{$\begin{array}{c}\text { human resource } \\
\text { development }\end{array}$} & \multicolumn{2}{|c|}{ Customer Focus } & \multicolumn{2}{|c|}{ Teamwork } & \multicolumn{2}{|c|}{ Total score } & \multirow{2}{*}{$\begin{array}{c}\text { Percent of } \\
\text { standards } \\
\text { ( national } \\
\text { assessment } \\
\text { ) } \\
\end{array}$} \\
\hline & $\mathrm{M}$ & SD & $\mathrm{M}$ & $\mathrm{SD}$ & $\mathrm{M}$ & SD & $\mathrm{M}$ & $\mathrm{SD}$ & $\mathrm{M}$ & $\mathrm{SD}$ & $\mathrm{M}$ & $\mathrm{SD}$ & \\
\hline Matini & 2.35 & 0.69 & 2.49 & 0.52 & 2.41 & 0.4 & 1.73 & 0.31 & 1.86 & 0.56 & $\begin{array}{c}2.17 \\
\% 43.4\end{array}$ & 0.34 & $\% 78$ \\
\hline Rajaee & 2.63 & 0.82 & 2.88 & 0.38 & 2.77 & 0.35 & 2.34 & 0.19 & 2.17 & 0.28 & $\begin{array}{c}2.56 \\
\% 51.1\end{array}$ & 0.3 & $\% 67$ \\
\hline Beheshti & 2.11 & 0.43 & 2.44 & 0.21 & 2.39 & 0.31 & 2.8 & 0.39 & 2.72 & 0.35 & $\begin{array}{c}2.49 \\
\% 49.8\end{array}$ & 0.27 & $\% 66$ \\
\hline Kargarnejad & 3.42 & 0.45 & 3.38 & 0.16 & 3.26 & 0.29 & 3.31 & 036 & 3.38 & 0.22 & $\begin{array}{l}3.35 \\
\% 67\end{array}$ & 0.06 & $\% 61$ \\
\hline Shohada & 2.86 & 0.81 & 4.24 & 0.27 & 3.97 & 0.34 & 3.66 & 0.28 & 3.63 & 0.4 & $\begin{array}{c}3.87 \\
\% 77.4\end{array}$ & 0.25 & $\% 60$ \\
\hline Naghavi & 2.69 & 0.58 & 2.98 & 0.17 & 3.05 & 0.29 & 2.75 & 0.11 & 3.03 & 0.39 & $\begin{array}{c}2.9 \\
\% 58\end{array}$ & 0.17 & $\% 56$ \\
\hline \multirow[t]{2}{*}{ Total } & $\begin{array}{c}2.9 \\
57.88 \\
\%\end{array}$ & 0.5 & $\begin{array}{c}3.1 \\
62.03 \%\end{array}$ & 0.19 & $\begin{array}{c}3.04 \\
\% 60.85\end{array}$ & 0.17 & $\begin{array}{c}2.8 \\
\% 56.35\end{array}$ & 0.1 & $\begin{array}{c}2.92 \\
58.41 \\
\%\end{array}$ & 0.29 & $\begin{array}{c}2.9 \\
\% 59.1\end{array}$ & 0.11 & $\% 64$ \\
\hline & \multicolumn{2}{|c|}{$<0.0001$} & \multicolumn{2}{|c|}{$<0.0001$} & \multicolumn{2}{|c|}{$<0.0001$} & \multicolumn{2}{|c|}{$<0.0001$} & \multicolumn{2}{|c|}{$<0.0001$} & & & \\
\hline
\end{tabular}

Based on results of table4, between comments of managers and supervisors in the hospitals there is a significant difference. (P-value <0.0001)

According to the above table, factor "management and leadership" in Kargarnejad Hospital has earned most points with mean (3.42) $68.4 \%$ and standard deviation 0.45. In contrast, Beheshti hospitals earned lowest score with mean (2.11) $42.2 \%$ and SD 0.43

Factor" organizational structure" in Shohada Hospital has earned most points with mean (4.24) $84.8 \%$ and standard deviation 0.27.In contrast, Beheshti hospitals earned lowest score with mean (2.44) $48.8 \%$ and SD 0.21

Factor "human resource development" in Shohada
Hospital has earned most points with mean (3.97) $79.4 \%$ and standard deviation 0.34. In contrast, Beheshti hospitals earned lowest score with mean (2.39)47.8\% and SD 0.31

Factor " Customer Focus " in Shohada Hospital has earned most points with mean (3.66) $73.2 \%$ and standard deviation 0.28.In contrast, Matini hospitals earned lowest score with mean (1.73)34.6\% and SD 0.31

Factor " Teamwork " in Shohada Hospital has earned most points with mean (3.63) $72.6 \%$ and standard deviation 0.4 .In contrast, Matini hospitals earned lowest score with mean (1.86 )37.2\% and SD 0.56

The second part of Table 4 is related to acquire assessment scores in the first round of the national 
assessment at accreditation program. As table shows, Matini hospitals earned most points with mean $78 \%$ and Naghavi hospital earned lowest score with mean $56 \%$.

Between Total score (comments of managers and supervisors) and Percent of standards there was no significant differences. $(\mathrm{P}$ value $=0.2$ a: 0.95 )

\section{Discussion}

In study of Kaplan \& et la (2010) leadership, organizational culture, basic data information systems and years involved in quality improvement were proposed as important to QI success. Other potentially important factors identified are included: physician involvement in QI, microsystem incentive to change, resources for QI, and QI team leadership.

The result study of zolfaghari \& kalanteri (2009) in university of Iran showed that Leadership and management, Employee, Strategic Planning, Communication, Organizational issues and Elements of Social-Cultural is the major obstacles to the implementation of TQM.

In the research of Torani and colleagues at hospital (2008) after five years have passed the implementation of Total Quality of Management, organizational culture, employee participation, human resources development, teamwork and leadership commitment can due to improvement and effectiveness of the services, and social acceptability of hospitals.

In this study, can be see the most important factor that must be considered to continue implementation of accreditation program is "Customer Focus". In this field the most important item that should be considered is: Planning for contact and communicate with clients and Planning for use applying comments of the customers.

Shohada and Kargarnejad hospital got a relative good point and Matini hospital got the lowest rating in this factor. In the study of Abasi and colleagues (2013) about standards of JCI showed, the lowest level of preparedness standards related to patient and family rights. In the research of Torani and colleagues (2008) about the classification factors influencing the implementation of quality management customer focus located in the first category with rankings relatively well.

According to comments of managers and supervisors another important factor that must be considered is" Leadership and management". The most important item of it is: Incentive mechanism for teams and staff in the program, Provide the human and financial resources to perform program and coherently follow up quality improvement activities.

Among the hospital of University, Kargarnejad hospital got a relative good point in this factor. Abasi and colleagues (2013),yarifard and colleagues ( 2012) Talib \& Zillur (2011), zolfaghari \& kalanteri (2009) have concluded that one of the major obstacles to the implementation of quality management is commitment and participation of leaders and managers.
The third factor in continue implementation of this program should be considered is" Teamwork". The most important item that should be addressed is: Use of quality control group for solve quality problems, Use of working groups to communicate and interact with clients and Encourage Individuals for teamwork by evaluation system.

Shohada and Kargarnejad hospital got a relative good point and Matini hospital got the lowest rating in this factor. In research of Torani and colleagues (2013), in the category of factors affecting implementation of TQM, teamwork was ranked fifth among the 8 factors .In study of Karimi and colleagues (2013) concluded that accreditation can be through setup and strengthening teams work and Strengthen communication and use of evidence-based decision will have beneficial effects. In study of Kilner \& Sheppard(2010),showed there is a positive relationship between high levels of staff satisfaction and team work.

The fourth factor is "human resource development". The main items of it is: Allocation of resources to educational requirements, Formulation and announcement of performance evaluation, Indicators and Understanding concepts and philosophy of program accreditation.

Shohada and Kargarnejad hospital got a relative good point and Matini\& Beheshti hospital got the lowest rating in this factor.

In the research of Frahbakhsh (2011) Concluded that improving performance of health care organizations using an integrated approach based on quality in addition to human resource development has a decisive role.In the study of zolfaghari \& kalanteri (2009) managing staff is one of the major barriers to deployment Of TQM. Yarifrd and colleagues (2012) in their study concluded that important barriers to accreditation can be lack of awareness and education at all levels of hospital staff. In the research of Torani and colleagues (2008) allocation of resources to educational requirements had the lower score among the human development items.

The last factor, that affecting implementation of accreditation program, is "organizational structure". In this field the main items that must be considered is: Delegation to employees in problem solving and Formulation of hospital structure according values.

Shohada \& Kargarnejad hospital got a relative good point and Matini\& Beheshti hospital got the lowest rating in this factor.In the study of zolfaghari \& kalanteri (2009), Torani and colleagues (2008) organizational issues that had been considered is: Lack of adequate delegation of authority, lack of attention to futures studies, Low adaptation structure with value of quality management.

Overall, according to survey of managers and supervisors of the University hospitals, Shohada Hospital, with $\% 77.4$ had the highest rating of the requirements for the accreditation program and Matini hospital with \%43.4 had the lowest rating. If that, according to national assessment, Matini hospital with $\% 78$ of total scores of accreditation program has ranked first among the six university hospital and Shohada hospital with \%60 rating 
has earned fifth place. Namely in Matini hospital between officials score and national assessment score there was $34 \%$ difference and $17 \%$ difference in Shohada hospital.

The lowest scores in the national assessment is related to Naghavi hospital with \%56 points that compared with hospital officials score (\%58) there is no much difference.

Second rank in the national assessment is related to Rajaee hospital with $\% 67$ points that compared with hospital officials score (\%51.1) there was $16 \%$ difference.

Third place rank in the national assessment is related to Rajaee hospital with $\% 66$ points that compared with hospital officials score (\%49.8) there was $17 \%$ difference.

Fourth place rank in the national assessment is related to Rajaee hospital with $\% 61$ points that compared with hospital officials score (\%67) there was $6 \%$ difference.

By comparing two scores had given, points of Matini, Rajaee and Beheshti hospital in the national assessment had greater than the rating officials and points of Shohada, Kargarnejad and Naghavi hospital in the national assessment had less than the rating officials.

But overall because there is no significant difference between the scores given by two groups ,can be said there is no great difficulty in definition and formulating accreditation standards. But in study of Yarmohamedian and etla (2014) suggests to authorities, study and plan for all aspects of the identified inconsistencies for successful implementation of this program in Iran and takes action to reduce or eliminate its effects through professional meetings and conferences in this field to find effective solutions.

\section{Conclusions}

This study focuses on requirements of the accreditation program. In the health sector, quality service and delivery in hospitals is one of the major problems. Hospital as one of the largest providers of health services have important role in the health of a society. High quality and standard of care helps the effectiveness, efficiency and success of this organization, so that without careful and systematic assessment and monitoring is not possible (Ghomri zareh \& etla, 2009)

Therefore, Iran's Ministry of Health has forced hospitals to implement the accreditation program in 2013.After the establishment the program and assessment by the Ministry of Health, researchers have tried to investigate obstacles and problems of the program.

Results showed for implementation of quality improvement programs, preparedness of studied hospitals is 59.1 percent.(with according important and necessary factors including: Leadership and management, Organizational structure, Human resource development, Customer focus, Team work) Among above factors, human resource development and organizational structure in good condition and customer focus, Leadership and management, team work in the medium status, are needed to reinforced and special attention.

By considering the results of this study and the importance of accreditation program is recommended:

1. Defined budget row for quality improvement programs.

2. Establish team reward and punishment system.

3. Assigned the responsibility of the patient to treatment team.

4. Communication skills training to employees for reform the existing culture

5. Pros and fee payments based on performance.

6. Formulation of Career path staff and managers.

7. Create a positive attitude among the evaluators to assess.

8. Because the accreditation program is in the initial stages of implementation, a complete revision is needed for definition and formulating standards.

\section{References}

[1] Abasi, s., tavakoli, n., \& moslehi, m. (2012). The preparedness of Hospitals with a Quality Management System based on the International Joint Commission accreditation standard functional model in. Health Information Management, 9(4), 502-512.

[2] Ahmadi, m., khoshgam, m., \& mohamad pour, a. (2008). Comparative study of $\mathrm{MOH}$ standards with international standards Joint Commission Accreditation. Hakim Research Journal, 10(4), 45- 52.

[3] Amerioun , A., Mahdavi , S., Mamaghani , H., \& etal. (2011). Assessment of International Joint Commission (IJC) accreditation standard in a military hospital laboratory. Iranian Journal of Military Medicine, 13(2), 75-80.

[4] Azami, s., tabrizi, s., \& etal. (2012). Knowledge and attitudes of managers about the accreditation of teaching hospitals, medical universities Tabriz and Ardebil. Journal of Health of Ardabil, 3(2), 7-15.

[5] Frahbakhsh, m., \& frahbakhsh, m. (2011). Review and comparative study of approaches to quality management in health care organizations. The first congress of the student's clinical governance and continuous quality improvement. Tabriz: Tabriz University of Medical Sciences.

[6] Ghaderi, s., \& hosseini, h. (2010). Model of factors affecting the quality of banking services. Vision for Business Management, 36(3), 89-115.

[7] Ghomri zareh, z., \& etal. (2009). The impact of the peer review process on the quality of nursing performance and patient satisfaction. Journal of Iranian Nursing, 22(62), 821.

[8] Guo, X., Duff, A., \& Hair, M. (2008). Service Quality Measurement in the Chinese Corporate Banking Market. International Journal of Bank Marketing, 26(5), 305-327.

[9] Kaplan, c., Brady, w., Dritz, c., \& etal. (2010). The Influence of Context on Quality Improvement Success in Health Care: A Systematic Review of the Literature. Milbank Quarterly, 88(4), 500-559.

[10] Karimi, s., shokri, a., \& etal. (2011). Influence of Accreditation in Services. The first congress of the student's clinical governance and continuous quality improvement. Tabriz: Tabriz University of Medical Sciences. 
12 Meghdad Rahati et al: Survey Requirements of the Accreditation Program after the First Round of the National Assessment at Hospital and Compared with the Results of It

[11] Kilner, E., \& Sheppard, L. A. (2010). The role of teamwork and communication in the emergency department: A systematic review. International Emergency Nursing, 18, $127-137$.

[12] Korrani, H., \& Mahdavi, M. (2008). A LOOK AT Hospital Accreditation Standards. Tehran: Ministry of Health \& Medical Education.

[13] Ministry of Health. (2007). Guideline and evaluation laws for general hospitals. tehran: Ministry of Health, Evaluation Office Publication.

[14] Talib, F., \& Zillur, R. (n.d.). Analysis of interaction among the barriers to total quality management implementation using interpretive structural modeling approach. International Journal of Services and Operations Management, 18(4), 563 - 587.

[15] Tofighi, S. (2002). Comparative study of health ministry standards for hospitals with ISO system and presenting appropriate model for general and special hospitals. tehran: Azad University.

[16] Torani, S., tabibi, s., \& shahbazi, B. (2008). Influencing factors on implementation of total quality management at teaching hospitals affiliated with Iran University of Medical Sciences. Hakim Research Journal, 11(2), 22- 32.

[17] Veillard, j., Champagne, F., \& etal. (2005). performance assessment framework for hospitals: the WHO regional office for Europe PATH project. Int J Qual Health Care, 17(6), 487-96.

[18] Yarifard, k., saadati, m., \& etal. (2012). Barriers and potential solutions to validate the performance of hospitals in Iran From the perspective of senior executives in Tabriz and Ardabil University of Medical Sciences. The first congress of the student's clinical governance and continuous quality improvement. Tabriz: Tabriz University of Medical Sciences.

[19] Yarmohammadian,M. Shokri,A. Bahmanziari,N. Kordi,A.(2014). The blind spots on Accreditation program. Journal of Health Systems Research,9(11)

[20] Zolfaghari, r., \& kalanteri, m. (2009). Barriers of TQM in Islamic Azad University,Unit Rudehen. New ideas on Educational Sciences, 3(2). 